\title{
A Novel Imaging Spectrometer for Energy-Distribution Measurements of Photoelectrons from GaAs Cathodes
}

\author{
C.D. Schröter, A. Rudenko, A. Dorn, R. Moshammer and J. Ullrich \\ Max-Planck-Institut für Kernphysik, 69029 Heidelberg, Germany
}

\begin{abstract}
The investigation of the photoelectron-escape mechanism from GaAs cathodes with negative electron affinity requires the detection of very low energy electrons. We have built a novel, UHV-compatible, spectrometer where the photoelectrons are imaged by a homogeneous electric field onto a position-sensitive detector. The time-of-flight of each single emitted electron and its position on the detector is measured. From these informations energy-distribution curves are extracted. The spectrometer has run successfully and preliminary energy-distribution curves have been measured. The system is now under improvement. With the optimized spectrometer, an excellent energy resolution (a few $\mathrm{meV}$ ) can be achieved.
\end{abstract}

\section{INTRODUCTION}

To investigate the photoelectron-escape mechanism from a photocathode with negative electron affinity, a spectrometer is required that allows the simultaneous measurement of the energy and angular distributions of very low energy electrons. In the past, measurements of the longitudinal energy distributions have been performed by several groups. Quite recently, complete energy distributions have been studied as a function of longitudinal and transverse energies. The employed method is based on selecting photoelectrons of a fixed longitudinal energy using a retarding field analyzer, and subsequently measuring the associated differential transverse energy distribution by applying an adiabatic magnetic compression technique [1].

Recently we have built up a novel imaging spectrometer which allows the simultaneous measurement of the longitudinal and transverse momenta for each individual photoelectron emitted from a GaAs surface [2]. Our spectrometer has run successfully and preliminary energy-distribution curves (EDC's) have been extracted from this information. However, the first tests have been performed under non-ideal conditions with high extraction fields and, hence, the thus far measured EDC's have lower resolution than what is ultimately achievable. Design changes have been made to be able to measure EDC's of photoelectrons with an improved energy resolution in the near future.

\section{EXPERIMENTAL SET-UP}

A schematic diagram of the spectrometer set-up is shown in figure 1 . The photocathode is illuminated by a short-pulsed laser diode (pulse width $\sim 100 \mathrm{ps}$ ). The laser focus spot size and its position on the cathode is controlled by a CCD camera. For the mea-

CP675, Spin 2002: $15^{\text {th }}$ Int'l. Spin Physics Symposium and Workshop on Polarized Electron

Sources and Polarimeters, edited by Y. I. Makdisi, A. U. Luccio, and W. W. MacKay (C) 2003 American Institute of Physics 0-7354-0136-5/03/\$20.00 
surements, highly doped p-type reflection mode GaAs/AlGaAs heterostructure crystals $\left(6 \times 10^{18} \mathrm{Zn} / \mathrm{cm}^{3}\right)$ are used with an active GaAs layer of $0.9 \mu \mathrm{m}$. For such a thin layer, the long tail of the electron pulse, generated by a $\delta$-pulse light excitation, extends out to less than 200 ps [3]. Hence, the photoelectron pulse width will be less than 300 ps and therefore short enough to not limit the final resolution of the electrons' time-of-flight (TOF) measurement. Details of the preparation technique of the photocathodes are described elsewhere $[2,4]$.

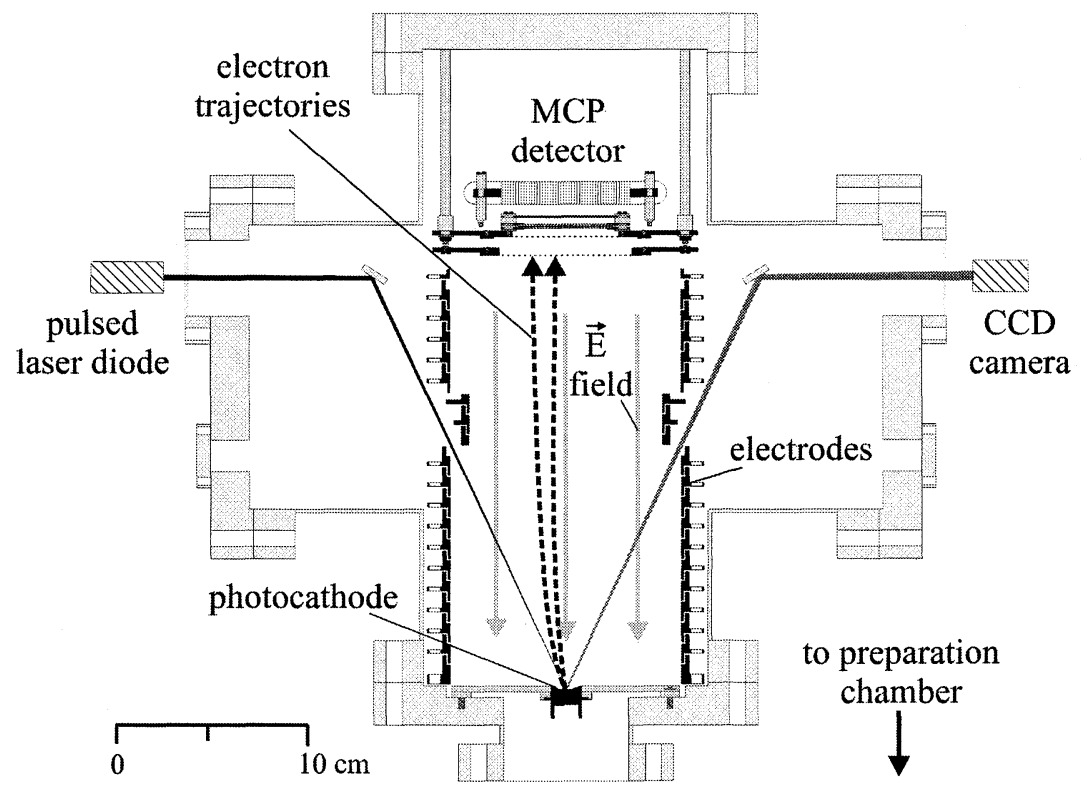

FIGURE 1. UHV chamber with spectrometer and MCP detector.

Emitted photoelectrons are projected by a homogeneous electric field onto a positionsensitive micro-channel plate (MCP) detector. The homogeneous field is produced by means of 20 cylindrical electrodes on which equidistant potentials are applied. The TOF of each single emitted photoelectron and its position on the detector are measured. From the TOF, the longitudinal momentum of the electron can be determined and from the position on the MCP detector, its transverse momentum can be extracted.

Computer simulations show that with the spectrometer an excellent energy resolution can be achieved (see figure 2). But in order to reach the highest resolution a very homogeneous electric field is required to guide the electrons to the detector. In the transverse direction, high resolution can be obtained even at high extraction fields; however, in the longitudinal direction, high resolution is achieved only for the smallest electric-field strengths. Then, even small electric and magnetic stray fields will influence the parabolic trajectories of the photoelectrons. Thus, for the whole apparatus, only UHV-compatible materials with very low permeability have been used. External fields are compensated by three pairs of Helmholtz coils installed pairwise, perpendicular to each other. The magnetic stray fields originating from ion getter pumps are shielded by $\mu$-metal housings. 

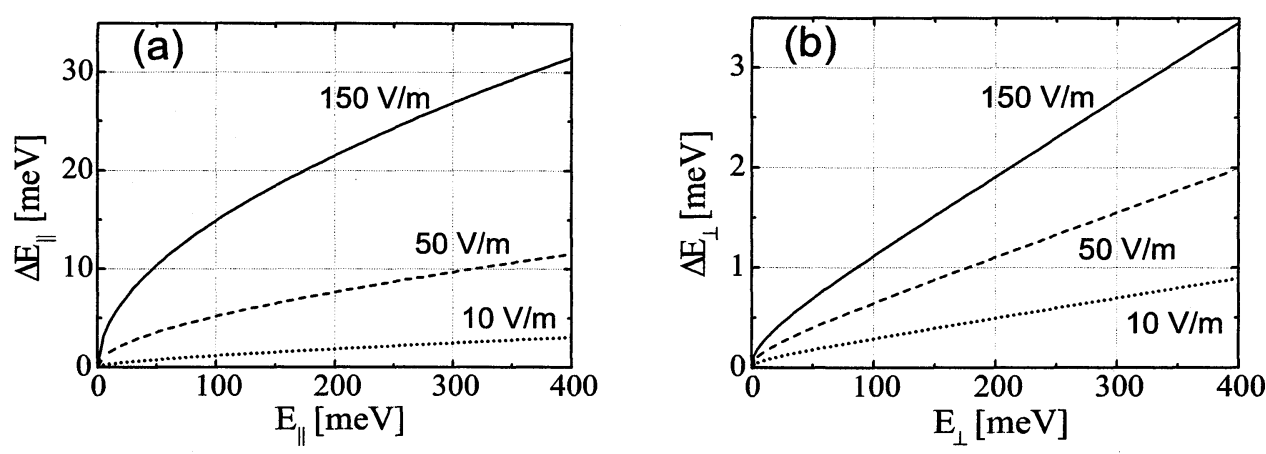

FIGURE 2. (a) Longitudinal and (b) transverse energy resolution of the spectrometer for electric field strengths of $150 \mathrm{~V} / \mathrm{m}, 50 \mathrm{~V} / \mathrm{m}$ and $10 \mathrm{~V} / \mathrm{m}$, respectively. The curves in (b) are plotted for $\mathrm{E}_{\|}=0 \mathrm{meV}$.

Great efforts have been undertaken to guarantee that the work functions of the different photocathode mounting components are homogeneous. Here, the path of a photoelectron is most sensitive to stray fields because the electron is not yet accelerated by the electric field. The potential of the photocathode mounts can be tuned relative to the potential of the first electrode of the spectrometer. This is important to minimize contactpotential differences in the photocathode mounting region. Moreover, the electrodes of the spectrometer are gold plated.

Using low-outgasing materials for all of the mounts, and pumping with ion getter pumps and volume getter strips should enable us to reach a vacuum base pressure in the $\sim 10^{-12}$ mbar range. This will ensure a long lifetime of the photocathodes and will lead to EDC measurements on undegraded cathodes in the near future.

\section{ACKNOWLEDGMENTS}

This work was partially supported by the Deutsche Forschungsgemeinschaft within the Leibniz-program. The heterostructure material was kindly put at our disposal by A. S. Terekhov. We are grateful to A. S. Terekhov as well as to D. A. Orlov and A. Wolf for their continuous support.

\section{REFERENCES}

1. Orlov D. A., Hoppe M., Weigel U.,Schwalm D., Terekhov A.S., and Wolf A., Appl. Phys. Lett. 78, 2721-2723 (2001).

2. Schröter C. D., Dorn A., Deipenwisch J., Höhr C., Moshammer R. and Ullrich J., International Workshop on Polarized Electron Source and Polarimeters, Nagoya 2000, in SPIN 2000, AIP Conference Proceedings 570, 996-999 (2001).

3. Hartmann P., Bermuth J., v. Harrach D., Hoffmann J., and Köbis S., Reichert E., Aulenbacher K., and Schuler J., Steigerwald M., J. Appl. Phys. 86, 2245-2249 (1999).

4. Schröter C.D., Dorn A., Moshammer R., Höhr C. and Ullrich J., International Workshop on Polarized Sources and Targets, Nashville 2001, Conference Proceedings, ed. by Derenchuk V.P. and von Przewoski B., (World Scientific, Singapore 2002), p. 166-169. 